\title{
DESENVOLVIMENTO DE UMA FERRAMENTA PARA O ENSINO DE TOPOGRAFIA
}

Daniel Ciro de Souza-danielciro6@gmail.com

Rafael Teixeirados Santos-rtxsanto@outlook.com

Rogério Rodrigues de Vargas - rogériovargas@unipampa.edu.br

Robert Martins da Silva - robertsilva@unipampa.edu.br

Alexandre Russini-alexandrerussini@unipampa.edu.br

Universidade Federal do Pampa - Laboratório de Sistemas Inteligentes e Modelagem

Rua Luiz Joaquim de Sá Britto, s/n Prómorar

9765000 - Itaqui - Rio Grande do Sul

Resumo: Os dispositivos móveis estão cada vez mais presentes no cotidiano, sendo considerado por muitos um item de primeira necessidade. Essa disseminação dos dispositivos móveis fez com que essa ferramenta fosse aplicada em distintas áreas. Na educação, não é diferente, o aparelho celular pode ser um forte instrumento e uma forma de metodologia a ser implantada pelo docente. Este trabalho mostra o desenvolvimento de uma ferramenta web que se adapte nos mais variados tipos de dispositivos com o objetivo de auxiliar o discente nas atividades propostas pelo professor na disciplina de topografia, mais especificamente nas realizações de cálculos referentes a levantamentos planimétricos de poligonais fechadas

Palavras-chave: Topografia. Poligonal Fechada. Educação. Dispositivos móveis

\section{INTRODUÇÃO}

Segundo Merije (2012), a União Internacional de Telecomunicações considera que o celular é a tecnologia mais rapidamente adotada na história. Isto é, dentre todas as tecnologias já criadas pelo homem os dispositivos móveis foram os que tiveram maior aceitação, sendo assim a tecnologia que mais disseminou-se celeremente no dia-a-dia da população. Com isto, Bastos (1997) defende a necessidade do estudo e aprofundamento de metodologias com dispositivos móveis nas escolas e universidades para usá-lo a favor do ensino. No entanto, em sala de aula muitas vezes o aparelho celular é responsável pela dispersão e distração no ensino. Isso faz com que os alunos não consigam manter um foco único, pois atenção é uma capacidade cerebral muito semelhante à concentração. Neste sentido, Seabra (2013) afirma que muitos professores prezam a proibição em relação ao uso de dispositivos móveis, tais como, celulares, tablets e notebooks em sala de aula, entretanto outros defendem a ideia que este pode ser um grande recurso didático e pedagógico agregando no aprendizado dos discentes caso seja aplicada uma metodologia correta. 
Mediante o exposto, o presente trabalho tem por objetivo a implantação de uma ferramenta exclusivamente desenvolvida para o ensino de topografia que se adapta a diversos dispositivos móveis, para auxiliar o discente no acompanhamento da disciplina de topografia, mais especificamente em levantamentos planimétricos de poligonais fechadas, ou seja, que sirva de instrumento para a interação durante o aprendizado, visto que em relação a dispositivos móveis na topografia, pouco se vê a utilização desta tecnologia principalmente na área da educação.

\section{MATERIAS E MÉTODOS}

O ambiente utilizado para desenvolvimento e testes da ferramenta, foi o notepad ++ . Esse ambiente é uma plataforma gratuita que abrange uma ampla variedade de linguagens de programação, além de possuir requisitos mínimos de instalação e processamento considerados baixos, como afirma Knight (2013). E as linguagens de programação utilizadas foram JavaScript para algumas funções de interação visual no navegador e PHP para elaboração dos cálculos no servidor. Além disso, para a estrutura básica visual da ferramenta, utilizou-se a linguagem de marcação HTML e a folha de estilo CSS. Segundo Atkinson e Gutmans (2000), a linguagem PHP é vista como vantajosa, pois permite que processos sejam realizados exclusivamente no servidor executando várias funções sem que altere a velocidade do mesmo, e é Open Source capaz de ser executado em diversos sistemas operacionais. De acordo com Flanagan (2011), a maioria dos sites e navegadores modernos usam JavaScript, em computadores de mesa, consoles de jogos e dispositivos móveis. Por conta desta compatibilidade, o JavaScript é a linguagem de programação lado do cliente, onde todo o processamento é realizado no dispositivo do usuário através do navegador, mais onipresente da história. Para Castro (2010) a linguagem de marcação HTML permite a formatação de texto, implantação de elementos gráficos, som e vídeo salvando tudo em um arquivo de texto que qualquer computador é capaz de ler.

A ferramenta, criada neste trabalho, detém a estrutura de desenvolvimento backend e frontend. A estrutura frontend, baseia-se em tudo o que é processado no lado do cliente, ou seja, no dispositivo do usuário sendo interpretado pelo navegador. Neste caso, o lado do cliente é responsável apenas pelo processamento do design, estruturas de layout e interação entre elas, além de ser responsável pela requisição com o servidor. Em contrapartida, o backend é toda informação processada no servidor, todo conteúdo que exige maior poder de processamento se concentra nesta estrutura. Este modelo de desenvolvimento, permite que a aplicação seja executada mais facilmente em diferentes tipos de aparelhos, não exigindo grande poder de processamento do dispositivo móvel, já que todos os cálculos para fechamento da poligonal são realizados pelo servidor. O layout foi desenvolvido de forma totalmente responsiva, ou seja, seu design se adapta automaticamente no dispositivo do usuário, conforme o tamanho de tela do aparelho, mantendo uma aparência agradável independente da plataforma utilizada.

\section{RESULTADOS}

A ferramenta divide-se em três partes: inserção dos dados iniciais, caminhamento por toda poligonal e impressão dos resultados, está disponível em um servidor web. No acesso do caminho de comunicação da ferramenta o usuário é direcionado para uma tela inicial composta por um menu dividido em três seções, Sobre, Créditos e Contato. Na parte inferior da tela, encontra-se o botão "próximo", onde o usuário pode iniciar o levantamento topográfico com um simples toque, conforme apresentado a seguir, na Figura 1: 
Figura 1 - Tela inicial da ferramenta TuTop

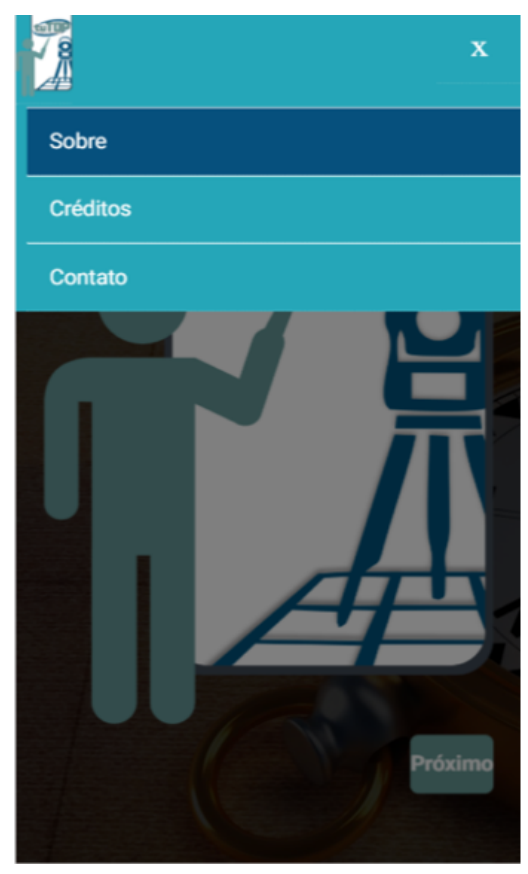

$\mathrm{Na}$ segunda tela (Figura 2), inicia-se o levantamento estacionando o aparelho no primeiro vértice da poligonal (E1), onde o usuário recebe as informações necessárias para o nivelamento e centralização do instrumento sobre o ponto topográfico.

Figura 2 - Tela de instruções para o nivelamento do aparelho.

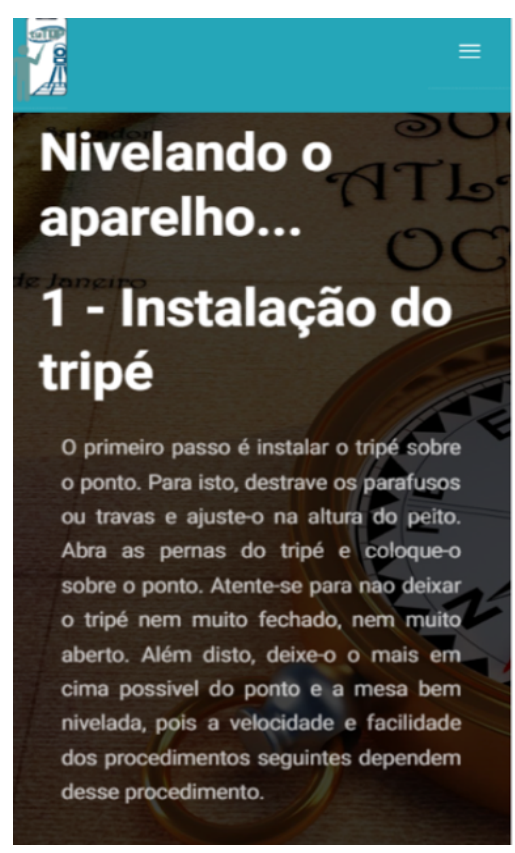

Em seguida, o usuário direciona-se para a terceira tela do aplicativo, a qual abrange todas as informações essenciais para o cálculo da poligonal fechada, como: número de vértices, sentido de caminhamento, azimute inicial, coordenadas iniciais e a precisão nominal do aparelho, necessárias para realizar o cálculo de fechamento e tolerância angular da poligonal, como mostrado na Figura 3. 
Figura 3 - Tela de instruções das informações iniciais da poligonal.

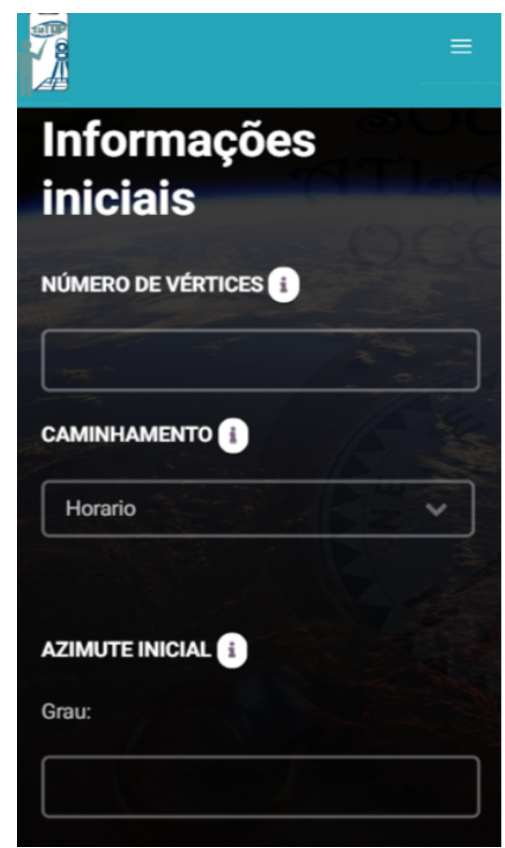

Nesta mesma tela, o usuário poderá recorrer a uma janela secundária ao premir nos ícones informativos. Esta janela secundária, tem como intuito dispor ao usuário determinadas informações complementares, auxiliando-o durante o levantamento e sanando possíveis dúvidas. Quando aberta, a janela de diálogo (Figura 4) bloqueia qualquer interação na janela principal, até que o diálogo seja encerrado.

Figura 4 - Janelas secundárias informativas (modais).

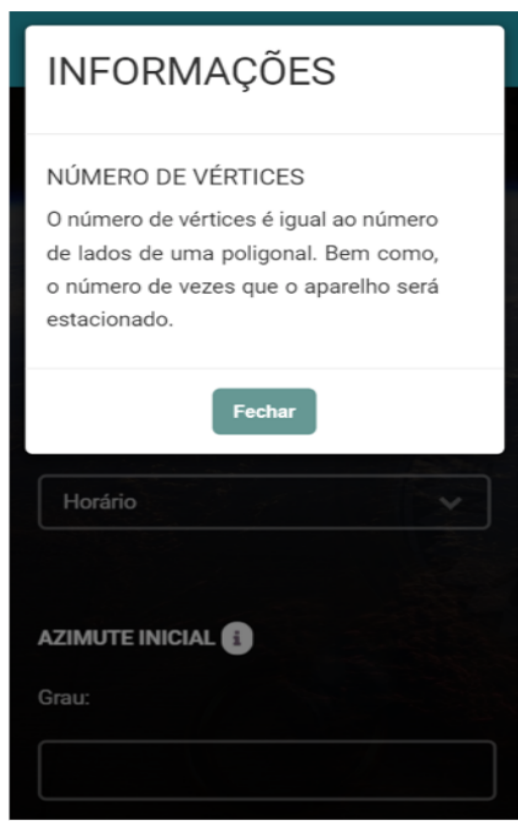

Definida as informações básicas, dá-se início ao caminhamento da poligonal. O usuário será instruído em qual vértice estacionar o aparelho, qual será a sua referência de ré e onde 
ocorrerá a sua visada de vante. Ao visar a ré, o aparelho é zerado, insere-se somente a distância horizontal. Na visada de vante, obtém-se o ângulo horizontal formado entre as visadas, além da distância horizontal medida entre a estação total e o vértice. Este processo, ocorrerá até que o usuário percorra por todos os pontos topográficos da poligonal, conforme mostra a Figura 5:

Figura 5 - Inserção dos dados coletados durante o caminhamento da poligonal

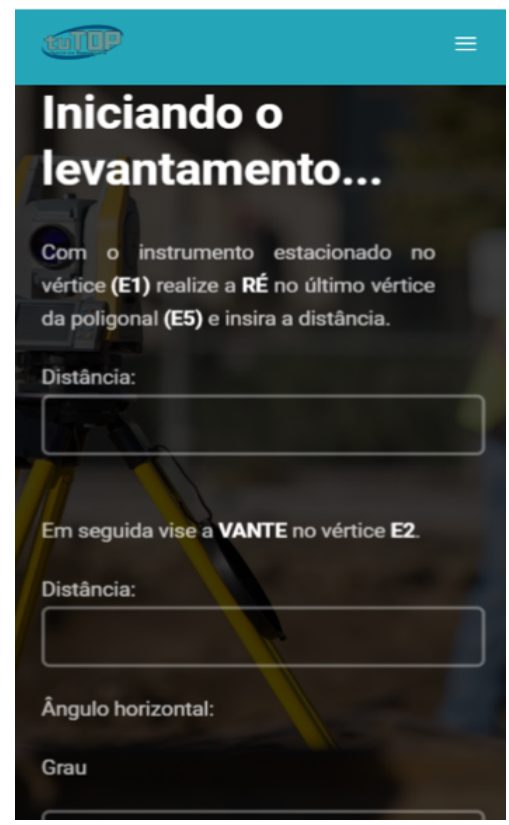

Após o usuário percorrer todo o caminhamento, basta prosseguir e o aplicativo retornará todos os cálculos da poligonal fechada, tais como, ângulos horizontais corrigidos, azimutes, coordenadas provisórias e finais, além de verificar a confiabilidade do levantamento, conforme exibido na Figura 6:

Figura 6 - Resultados finais da poligonal.

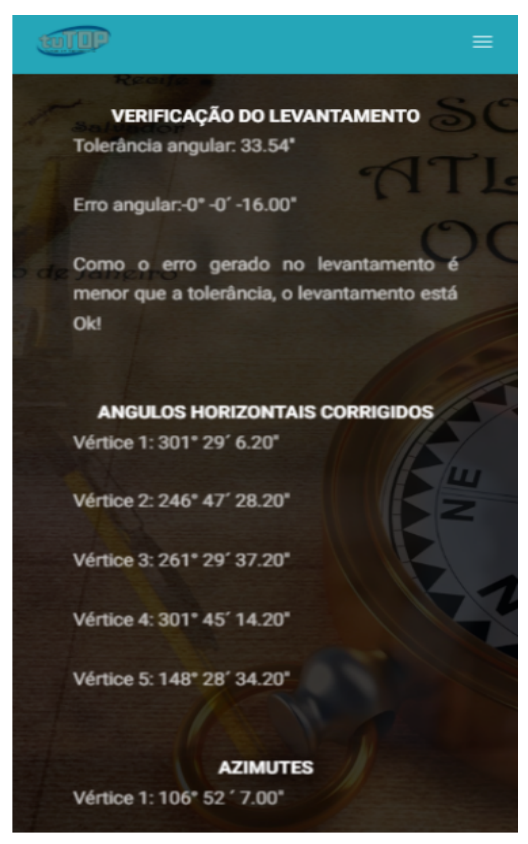




\subsection{Validação da ferramenta}

Caso h A seguir apresentam-se através das Tabelas 1 a 5, os resultados dos cálculos obtidos manualmente e por meio da ferramenta TuTop, conforme os dados de um levantamento planimétrico de poligonal fechada (Figura 7):

Figura 7 - Croqui de uma poligonal fechada.

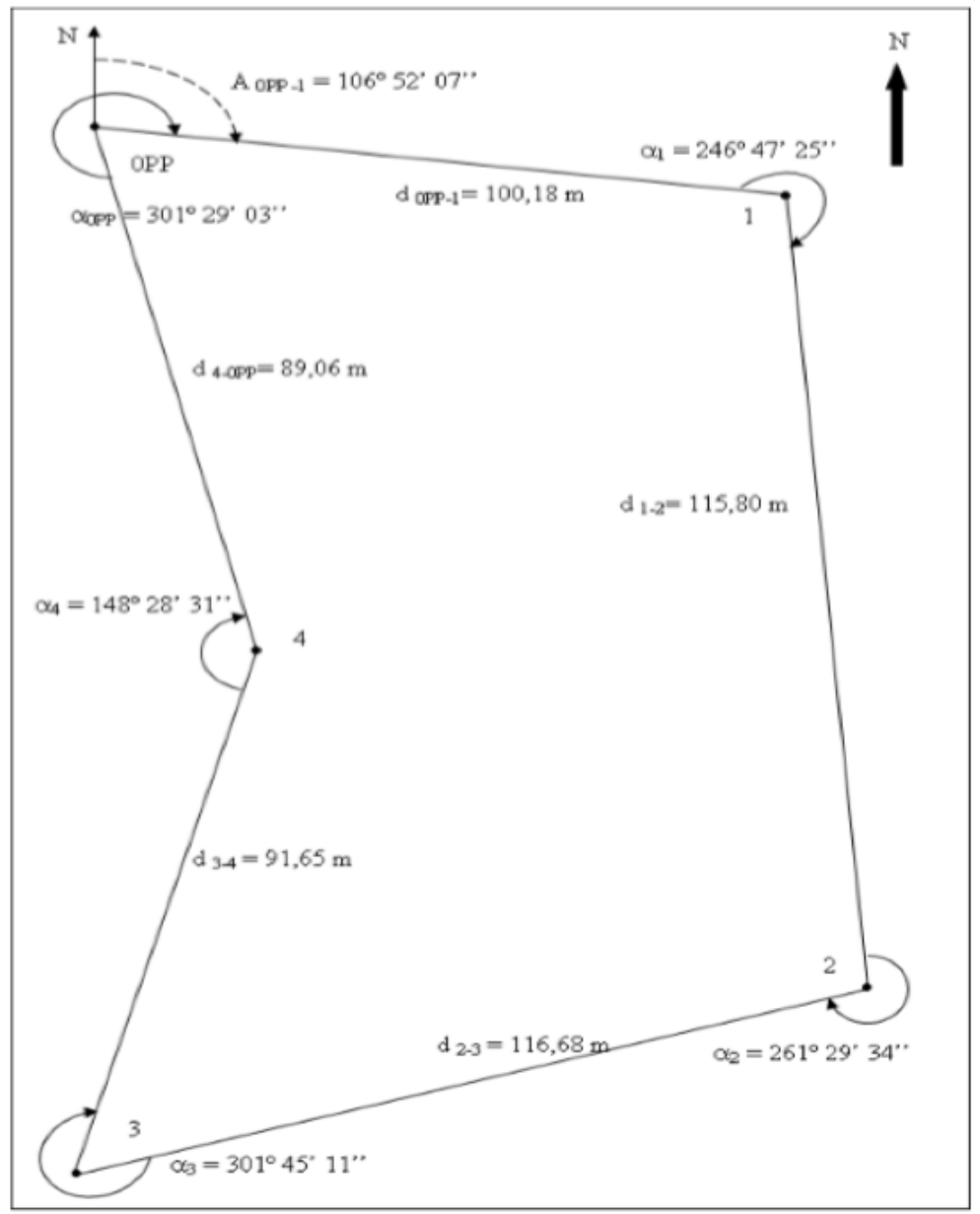

Fonte: Veiga, Koenig e Zanetti (2007).

Tabela 1 - Erro angula e tolerância angular calculadas manualmente e através do TuTop

\begin{tabular}{c|c|c|}
\hline & TuTop & Manual \\
\hline Erro angula (") & -16 & -16 \\
\hline Tolerância angular (“) & $+/-22$ & $+/-22$ \\
\hline
\end{tabular}

Tabela 2 - Azimutes calculados pelo TuTop Cálculo dos Azimutes (TuTop)

\begin{tabular}{c|c|c|l|}
\hline Ponto & Direção & Ângulo Corrigido & \multicolumn{1}{|c|}{ Azimute } \\
\hline OPP & OPP-1 & - & $106^{\circ} 53^{\prime} 07^{\prime}$ \\
\hline 1 & $1-2$ & $246^{\circ} 47^{\prime} 28,20^{\prime}$ & $173^{\circ} 39^{\prime} 35,20^{\prime}$ \\
\hline 2 & $2-3$ & $262^{\circ} 29^{\prime} 37,20^{\prime}$ & $255^{\circ} 9^{\prime} 12,40^{\prime \prime}$ \\
\hline 3 & $3-4$ & $301^{\circ} 45^{\prime} 14,20^{\prime}$ & $16^{\circ} 54^{\prime} 26,60^{\prime}$ \\
\hline 4 & $4-\mathrm{OPP}$ & $148^{\circ} 29^{\prime} 06,20^{\prime}$ & $345^{\circ} 23^{\prime} 0,80^{\prime \prime}$ \\
\hline $5=$ OPP & & $301^{\circ} 29^{\prime} 06,20^{\prime}$ & $106^{\circ} 52^{\prime} 07^{\prime}$ \\
\hline
\end{tabular}


Tabela 3 - Azimutes calculados manualmente

\begin{tabular}{c|c|c|c|}
\hline \multicolumn{4}{|c|}{ Cálculo dos Azimutes (Manual) } \\
\hline Ponto & Direção & Ângulo Corrigido & Azimute \\
\hline OPP & OPP-1 & - & $106^{\circ} 53^{\prime} 07^{\prime \prime}$ \\
\hline 1 & $1-2$ & $246^{\circ} 47^{\prime} 28,20^{\prime \prime}$ & $173^{\circ} 39^{\prime} 35,20^{\prime}$ \\
\hline 2 & $2-3$ & $262^{\circ} 29^{\prime} 37,20^{\prime \prime}$ & $255^{\circ} 9^{\prime} 12,40^{\prime \prime}$ \\
\hline 3 & $3-4$ & $301^{\circ} 45^{\prime} 14,20^{\prime \prime}$ & $16^{\circ} 54^{\prime} 26,60^{\prime \prime}$ \\
\hline 4 & $4-\mathrm{OPP}$ & $148^{\circ} 29^{\prime} 06,20^{\prime \prime}$ & $345^{\circ} 23^{\prime} 0,80^{\prime \prime}$ \\
\hline $5=\mathrm{OPP}$ & & $301^{\circ} 29^{\prime} 06,20^{\prime \prime}$ & $106^{\circ} 52^{\prime} 07^{\prime}$ \\
\hline
\end{tabular}

Tabela 4 - Coordenadas provisórias calculadas manualmente e através do Tutop

\begin{tabular}{c|c|c|c|c|c}
\hline \multicolumn{2}{c}{ Coordenadas provisória (TuTop) } & \multicolumn{3}{c|}{ Coordenadas provisórias (Manual) } \\
\hline Ponto & $\mathrm{X}(\mathrm{m})$ & $\mathrm{Y}(\mathrm{m})$ & Ponto & $\mathrm{X}(\mathrm{m})$ & $\mathrm{Y}(\mathrm{m})$ \\
\hline OPP & 224,19 & 589,25 & OPP & 224,19 & 589,25 \\
\hline 1 & 320,06 & 560,18 & 1 & 320,05 & 560,17 \\
\hline 2 & 332,85 & 445,09 & 2 & 332,84 & 445,08 \\
\hline 3 & 220,07 & 415,19 & 3 & 220,06 & 415,19 \\
\hline 4 & 224,19 & 502,91 & 4 & 246,71 & 502,87 \\
\hline $5=$ OPP & 224,25 & 589,06 & $5=$ OPP & 224,24 & 589,06 \\
\hline
\end{tabular}

Tabela 5 - Coordenadas corrigidas calculadas manualmente e através do TuTop

\begin{tabular}{c|c|c|c|c|c|}
\multicolumn{2}{c}{ Coordenadas corrigidas (TuTop) } & \multicolumn{3}{c|}{ Coordenadas corrigidas (Manual) } \\
\hline Ponto & $\mathrm{X}(\mathrm{m})$ & $\mathrm{Y}(\mathrm{m})$ & Ponto & $\mathrm{X}(\mathrm{m})$ & $\mathrm{Y}(\mathrm{m})$ \\
\hline OPP & 224,19 & 589,25 & OPP & 224,19 & 589,25 \\
\hline 1 & 320,05 & 560,22 & 1 & 320,05 & 560,21 \\
\hline 2 & 332,84 & 445,13 & 2 & 332,83 & 445,12 \\
\hline 3 & 220,06 & 415,23 & 3 & 220,05 & 415,23 \\
\hline 4 & 224,19 & 502,91 & 4 & 246,70 & 502,92 \\
\hline $5=$ OPP & 224,19 & 589,25 & $5=$ OPP & 224,24 & 589,25 \\
\hline
\end{tabular}

Os resultados demonstram-se satisfatórios no levantamento planimétrico de uma poligonal fechada conforme mostrado nas tabelas anteriores. Observe que há uma pequena diferença de valores nas casas decimais, isso ocorre devido a alguns arredondamentos.

\section{CONCLUSÃO}

Com o auxílio desta tecnologia e com o conhecimento absorvido em sala de aula através do docente, espera-se que a ferramenta TuTop proporcione maior dinamismo nas aulas de campo que em algumas ocasiões tem um número elevado de matriculados. Há uma enorme dificuldade por parte dos docentes em auxiliar todos de maneira atenciosa, pois são impossibilitados de sanar todas as dúvidas e dificuldades que surjam durante um levantamento topográfico de maneira eficiente. Esta ferramenta auxiliará o discente a realizar o levantamento sendo guiado em todas as etapas da poligonal, com janelas informativas em caso de dúvidas, obtenção de resultados dos cálculos e ao final do levantamento verificar a precisão alcançada. Além disso, em alguns testes realizados em campo e laboratório com um pequeno grupo envolvendo exalunos da disciplina de topografia, foi possível não apenas usufruir da ferramenta para levantamentos em campo, mas também para a resolução de exercícios propostos, interpretando 
a poligonal, seu caminhamento e informações disponibilizadas, inserindo estes valores na ferramenta e comparando o resultado com os obtidos manualmente. Porém ainda não foram realizados testes a longo prazo com os discentes.

Como possíveis trabalhos futuros, pode-se apontar o desenvolvimento de um aplicativo para as plataformas dos sistemas operacionais Android e iOS, também disponíveis de forma gratuita. Porém com a possibilidade de se trabalhar off-line. Além disto, haverá um estudo maior junto aos discentes da disciplina de topografia afim de obter um feedback por parte deles.

\title{
REFERÊNCIAS
}

ATKINSON, Leon. Core PHP programming: Using PHP to build dynamic web sites. Upper Saddle River, NJ: Prentice Hall. 2000.

BASTOS, J. A. de S. A. Educação e Tecnologia. Revista Técnico- científica dos Programas de Pós-Graduação em Tecnologia dos CEFETs PR/MG/RJ, Curitiba, CEFET- PR, n. 1, abr. 1997. CASTRO, Elizabeth. HTML, XHTML \& CSS. 6a edição, Rio de Janeiro: Alta Books, 2010.

FLANAGAN, David. JavaScript: The Definitive Guide. 6a edição, United States of America: O’Reilly, 2011.

KNIGHT, Jonathan. Notepad++ User Manual. UNT, 2013.

MERIJE, Wagner. Mobimento: Educação e comunicação mobile. São Paulo: Editora Peirópolis, 2012.

SEABRA, C. O Celular na Sala de Aula. Educação em Revista. Sindicato do Ensino Privado. SINEPE. Rio Grande do Sul, ed. 96, março de 2013.

VEIGA, Luis Augusto Koenig; ZANETTI, Maria Aparecida Zehnpfenning; FAGGION, Pedro Luis. Fundamentos de Topografia. UFPR: Curitiba, 2007.

\section{DEVELOPMENT OF A TOOL FOR TOPOGRAPHY TEACHING}

\begin{abstract}
Mobile devices are increasingly present in our daily lives and are considered a necessary item by many people. As such, mobile devices have been used successfully in different areas. In education, mobile phones can be a useful tool that professors can apply during classes. The present study discusses the development of a web application that aims to assist students in topography classes. More specifically, the tool makes calculations used in planimetric surveys of closed polygonal areas easier. As a web application, the proposed tool adapts to many types of devices, regardless of operational system, screen size, and other specific aspects, which facilitates its use among students.
\end{abstract}

Keywords: Topography. Polygonal Traverse. Education. Mobile Devices. 